\title{
GIẢI PHÁP TÌM KIẾM NGŨ̃ NGHĨA CHO VĂN BẢN KHOA HỌC MÁY TÍNH TIẾNG VIẸT
}

\author{
Nguyễn Hữu Vĩnh ${ }^{a^{*}}$
}

${ }^{a}$ Khoa Công nghệ Thông tin, Trương Đại học Thủ Dầu Một, Bình Duơng, Việt Nam

Lịch sử bài báo

Nhận ngày 04 tháng 11 năm 2016 | Chỉnh sửa ngày 11 tháng 04 năm 2017

Chấp nhận đăng ngày 11 tháng 05 năm 2017

\section{Tóm tắt}

Bài báo này tập trung nghiên cúu vấn đề tìm kiếm ngũ nghĩa. Mục đỉch của chúng tôi là tìm hiểu và xây dưng công cu tìm kiếm theo ngũ nghĩa để có thể tìm kiếm thông tin chính xác và đầy đủ, để hạn chế phần nào tìm kiếm theo tù khóa của các hệ thống tìm kiếm hiện tại. Xây dựng mô hình tổ chức, luu trũ, quản lý các tài liệu về công nghệ thông tin tiếng Việt trong 3 lĩnh vưc chính là: Phần mềm; Lập trình; và Mạng máy tính trên các máy tính hố trọ tìm kiếm theo ngũ nghĩa. Xây dưng mô hình quản lý cơ sở tài liệu có ngũ nghĩa trong đó có biểu diễn ngũ nghĩa liên quan đến nội dung tài liệ; Xây dụng các giải pháp, kỹ thuật biểu diễn và xủ lý trong tìm kiếm tài liệu.

Từ khóa: Ontology; Search engine; Semantic document.

\section{GIỚI THIỆU}

Ngày nay, hầu hết các hệ thống tìm kiếm điều theo hướng truyền thống là tìm theo từ khóa. Theo cách tìm kiếm này, khi ta gõ vào từ cần tìm, hệ thống tìm kiếm sẽ hiển thị các tài liệu mà trong đó chứa từ khóa cần tìm. Do đó, kết quả trả về là một danh sách các tài liệu, mà có thể tài liệu này không liên quan gì đến nội dung ta cần tìm. Và đôi khi hệ thống này không đưa ra kết quả chính xác, tức là thừa tài liệu không cần thiết nhưng thiếu tài liệu liên quan. Vấn đề đặt ra là phải xây dựng một hệ thống tìm kiếm để khắc phục hiện trạng nêu trên. Để giải quyết vấn đề này, ta cần xây dựng hệ thống tìm kiếm đầy đủ thông tin mà người dùng mong muốn. Nghĩa là hệ thống tìm kiếm ngữ nghĩa dựa trên thông tin người dùng nhập vào. Trong nghiên cứu này, chúng tôi xây dựng Ontology để có thể biểu diễn ngữ nghĩa và tìm kiếm ngữ nghĩa trong phạm vi ứng dụng cụ thể "Tìm kiếm nội dung tài liệu dựa trên ngữ nghĩa, ứng dụng vào việc tìm kiếm văn bản khoa học 
máy tính (cho các tài liệu tiếng Việt)". Với mục đích tìm hiểu và xây dựng công cụ tìm kiếm theo ngữ nghĩa để có thể tìm kiếm thông tin chính xác và đầy đủ, hạn chế phần nào tìm kiếm theo từ khóa của các hệ thống tìm kiếm hiện tại.

\section{TỔNG QUAN VỀ TÌM KIẾM THÔNG TIN VÀ TRUY TÌM NGŨ NGHĨA}

\subsection{Vấn đề tìm kiếm thông tin}

Ngày nay máy tính đã được sử dụng trong mọi lĩnh vực của đời sống. Trong lĩnh vực lưu trữ dữ liệu, chỉ cần ổ cứng 10x15x3 cm là đã có thể lưu trữ được số lượng sách tương ứng với cả thư viện sách khổng lồ. Vì vậy kho thông tin trong máy tính tăng trưởng không ngừng và thật khó khăn cho công tác tìm kiếm (nhất là tìm kiếm trên các tài liệu). Hãng Microsoft đã hỗ trợ tìm kiếm tự động bằng công cụ tìm kiếm được tích hợp sẵn trong hệ điều hành Windows, trong đó cho ta hai cách thức tìm kiếm tập tin là tìm theo từ khoá tên tập tin (All or part of the file name) đưa ra các tập tin có tên chứa khoá tìm kiếm và tìm theo từ khoá nội dung trong tập tin (A word or phrase in the file) đưa ra nội dung các tập tin có chứa một từ hoặc cụm từ giống với từ khoá. Mặc dù tìm kiếm trong Windows hỗ trợ mạnh chức năng tìm kiếm theo tên tập tin, nhưng tìm theo nội dung trong tập tin vẫn còn có những hạn chế nhất định. Chẳng hạn, tìm kiếm chỉ đưa ra các tập tin có chứa chính xác từ khoá tìm kiếm, như vậy sẽ rất khó khăn nếu người dùng không nhớ chính xác từ khoá có trong nội dung tài liệu mà chỉ nhớ gần đúng với từ khoá, hơn nữa công cụ tìm kiếm không chỉ ra được cụm từ khoá tìm được nằm ở đâu trong văn bản và các tài liệu có liên quan về mặt ngữ nghĩa. Nếu cần người dùng lại một lần nữa phải đi dò tìm bằng các công cụ tìm kiếm khác.

Hiện tại đã có một số nghiên cứu về lĩnh vực tìm kiếm theo ngữ nghĩa. Nghiên cứu của Christopher, Prabhakar, và Hinrich (2008) đã dùng symbolic link cho hệ thống cấp bậc dựa trên thuộc tính - giá trị cho hệ thống chỉ mục tự động, việc tìm kiếm thuận lợi dựa trên ý tưởng các thư mục ảo. Nội dung của các thư mục ảo này là kết quả của truy vấn được tạo thông qua hệ thống phân cấp, bài báo này cũng là tiền đề phát sinh ra các hệ thống tập tin ngữ nghĩa (Semantic File System - SFS) và các công cụ tìm kiếm trên Desktop (Desktop search) ngày nay. Nghiên cứu của Christopher và ctg. (2008) chủ yếu 
dựa trên các kết quả nghiên cứu của Stokoe, Oakes, và Tait (2003). Tại Việt Nam, dự án của Ngô (2007) triển khai cách thức tạo SFS với sự kết hợp của Ontology. Tác giả cho thấy rằng sự kết hợp hệ thống tập tin với Ontology là mục tiêu lý tưởng để tạo ra một SFS mang nhiều ngữ nghĩa hơn vì ngữ nghĩa có được là sự so khớp khái niệm chứ không phải so khớp chuỗi theo như các cách thức đã có. Mỗi khái niệm sẽ có mối quan hệ ngữ nghĩa đi kèm với nó để biết các khái niệm liên quan như hệ thống tập tin được tổ chức là có ngữ nghĩa và ngữ nghĩa sẽ phụ thuộc vào cách Ontology liên kết với nó. Trương (2009) nghiên cứu trên lĩnh vực khoa học máy tính với ngôn ngữ tiếng Anh, dò tìm trên các dạng tập tin pdf, html, txt. Tác giả cũng nêu được vấn đề quan hệ nghĩa hẹp và nghĩa rộng của tài liệu. Các kết quả nghiên cứu hiện nay còn rất hạn chế trong việc xử lý ngữ nghĩa, tổ chức và xử lý tích hợp dữ liệu, thông tin, tri thức. Việc cập nhật các tài liệu thông tin dựa trên không chỉ các từ khóa (dữ liệu), mà còn dựa trên ngữ nghĩa nội dung hay tri thức liên quan.

\subsection{Vấn đề truy tìm ngữ nghĩa}

Hiện nay, hầu hết các hệ thống tìm kiếm thông tin (information retrieval - hệ thống IR) thực chất chỉ là hệ thống tìm kiếm tài liệu (document retrieval). Nghĩa là hệ thống tìm trong số các tài liệu trong cơ sở dữ liệu lưu trữ, tài liệu nào có nội dung liên quan đến nhu cầu thông tin người dùng. Sau đó người dùng sẽ tìm kiếm thông tin họ cần trong các tài liệu liên quan đó. Chỉ có một vài hệ thống lập chỉ mục cho các đơn vị như câu, đoạn, trang văn bản, và xem những đơn vị đó như một tài liệu nhỏ (Wood, 1997).

Các hệ thống tìm kiếm hiện nay phần lớn vẫn dựa trên từ khóa. Một danh sách các từ khóa là dạng biểu diễn sơ lược nhất của nội dung, nghĩa là mỗi tài liệu được biểu diễn bởi một tập từ hay cụm từ được rút trích từ chính nội dung của tài liệu và do đó, cách biểu diễn này mang mức độ thông tin thấp nhất. Mối quan hệ ngữ nghĩa giữa các từ khóa hay nghĩa của các từ, cụm từ không được xét đến. Đặc biệt đối với người sử dụng ít kinh nghiệm thì khó có thể đặc tả đúng từ khóa cho vấn đề cần tìm kiếm. Đó chính là lý do cơ bản khiến cho các hệ thống tìm kiếm hiện nay có kết quả trả về không phải lúc nào cũng thỏa mãn yêu cầu tìm kiếm của người sử dụng, như là độ chính xác không cao khi kết quả trả về quá nhiều mà tỷ lệ số tài liệu hữu ích trên tổng số tài liệu trả về thấp. 
Từ những mô hình tìm kiếm đơn giản ban đầu như Boolean, nhiều tác giả đã nỗ lực cải thiện hiệu quả của việc tìm kiếm thông qua các mô hình phức tạp hơn như mô hình không gian Vector, các mô hình xác suất (probabilitic models), mô hình ngôn ngữ (language model), Latent Semantic Indexing Model (Christopher và ctg., 2008). Ngoài ra, nhiều tác giả còn sử dụng thuật ngữ (term) là các từ (word) hay cụm từ (phrase) thay vì chỉ dùng từ. Hệ thống đã cho kết quả chính xác hơn khi sử dụng thuật ngữ để tìm kiếm theo một miền nhất định nào đó. Nhiều nghiên cứu khác nhằm nỗ lực thay đổi cách đánh trọng số, đưa vào xử lý ngôn ngữ tự nhiên (David, 2008), khử nhập nhằng (Stokoe và ctg., 2003), mở rộng tài liệu, mở rộng câu truy vấn (Aly, 2008), cũng góp phần làm tăng hiệu quả tìm kiếm. Mặc dù có nhiều cải tiến để cải thiện kết quả, những hạn chế của việc sử dụng từ khóa vẫn chưa được khắc phục.

Hiện nay trong lĩnh vực của khoa học máy tính có một sự chuyển hướng dần đến những thứ mà có thể gọi là sự hướng tri thức (knowledge orientation) hoặc xử lý ngữ nghĩa. Theo đó, những hệ thống tìm kiếm dựa trên khái niệm (concept) được nghiên cứu phát triển nhằm thay thế cho những hệ thống truyền thống vốn đã bộc lộ nhiều khuyết điểm lớn, việc tìm kiếm sẽ dựa trên không gian các khái niệm và các mối quan hệ ngữ nghĩa giữa chúng. Những cách tiếp cận theo hướng ngữ nghĩa hay theo cấu trúc khái niệm này hướng tới việc mô phỏng một cách tự nhiên cách con người giao tiếp, nghĩa là mô phỏng cấp độ hiểu về ý nghĩa của từ, cụm từ hay văn bản mà người dùng cung cấp tương ứng với những gì người dùng nghĩ. Tuy nhiên, việc xây dựng một hệ thống truy tìm tài liệu dựa trên khái niệm, có biểu diễn và xử lý ngữ nghĩa trong tìm kiếm cho đến nay vẫn còn là vấn đề rất khó. Hệ thống phải sử dụng đến tri thức về một lĩnh vực nào đó và việc lập chỉ mục phải dựa vào một cấu trúc khái niệm phân lớp có sẵn, đặc biệt, vấn đề nghiên cứu xây dựng mô hình biểu diễn tài liệu giàu ngữ nghĩa hơn là một bước tiền xử lý rất quan trọng. Nhằm khắc phục những điểm yếu trong việc biểu diễn tài liệu theo các mô hình truyền thống như mô hình túi từ (bag of words), mô hình không gian vector, các mô hình biểu diễn không ngừng được nghiên cứu phát triển, hàm chứa được nhiều hơn những suy nghĩ mà con người muốn diễn đạt, đồng thời nâng cao hiệu quả sử dụng, trong đó nổi bật nhất là các mô hình đồ thị như mạng ngữ nghĩa, đồ thị khái niệm (Concept Graph - 
$C G s)$, CGs cải tiến, đồ thị hình sao, đồ thị tần số, đồ thị khoảng cách, đồ thị song phương, được đánh giá có nhiều tiềm năng, vì tận dụng được các thông tin quan trọng về cấu trúc và các mối quan hệ ngữ nghĩa vốn không được xét đến trong các mô hình truyền thống. Các nghiên cứu về tìm kiếm theo ngữ nghĩa hiện nay chủ yếu tập trung cải thiện hiệu quả tìm kiếm theo các hướng chính như: Nghiên cứu việc khai thác những nguồn tri thức như WordNet, UMLS; Nghiên cứu việc mở rộng tài liệu và mở rộng câu truy vấn; Nghiên cứu việc sử dụng các kỹ thuật khác để hỗ trợ quá trình tìm kiếm như xử lý ngôn ngữ tự nhiên, fuzzy, khử nhập nhằng, phân loại, hay các kỹ thuật để sắp xếp kết quả tìm kiếm; và Nghiên cứu cách thức xây dựng, biểu diễn và so khớp các cấu trúc khái niệm, các cách lập chỉ mục khái niệm (Woods, 1997).

Hiện nay, cách tiếp cận cho việc biểu diễn ngữ nghĩa dựa trên các Ontology được xem là cách tiếp cận hiện đại và phù hợp nhất cho việc thiết kế biểu diễn, xử lý nội dung và ý nghĩa của các tài liệu của con người. Việc sử dụng Ontology và từ điển từ vựng làm tăng độ chính xác và khả năng vét cạn trong quá trình tìm kiếm thông tin theo hướng ngữ nghĩa. Ontology là bản mô tả tường minh các khái niệm trong một miền ứng dụng nào đó và quan hệ giữa những khái niệm này cùng một số luật logic và suy diễn, cho phép suy luận khái niệm mới từ các khái niệm đã có. Ontology cung cấp từ vựng thống nhất cho việc trao đổi thông tin giữa các ứng dụng. Hiện nay đã có nhiều nghiên cứu xây dựng các hệ thống hỗ trợ giáo dục theo cách tiếp cận sử dụng Ontology được đề cập trong nghiên cứu của Cao (2001); Christopher và ctg. (2008); Lê, Từ, và Huỳnh (2006); và Nguyễn và Hoàng (2009).

\section{CÁC KHÁI NIỆM LIÊN QUAN}

\subsection{Hệ thống IR}

Một hệ thống IR gồm 3 thành phần: Dữ liệu đầu vào (input); Bộ xử lý (processor); và dữ liệu đầu ra (output), được thể hiện như Hình 1.

Phần Input: Đầu vào thể hiện cho mỗi (tài liệu, truy vấn) thích hợp để máy tính sử dụng. Tài liệu đại diện (document representative) có thể là danh sách các từ (word) được trích xuất mà nó có ý nghĩa, nói chính xác đó là máy tính xử lý ngôn ngữ tự nhiên, hoặc là dùng ngôn ngữ nhân tạo để diễn đạt các truy vấn và tài liệu. Khi hệ thống truy tìm 
trực tuyến, thì người dùng có thể thay đổi trong suốt quá trình tìm kiếm. Vì thế có nhiều kỳ vọng về sự cải thiện sự thực thi truy tìm trong các lần kế tiếp.

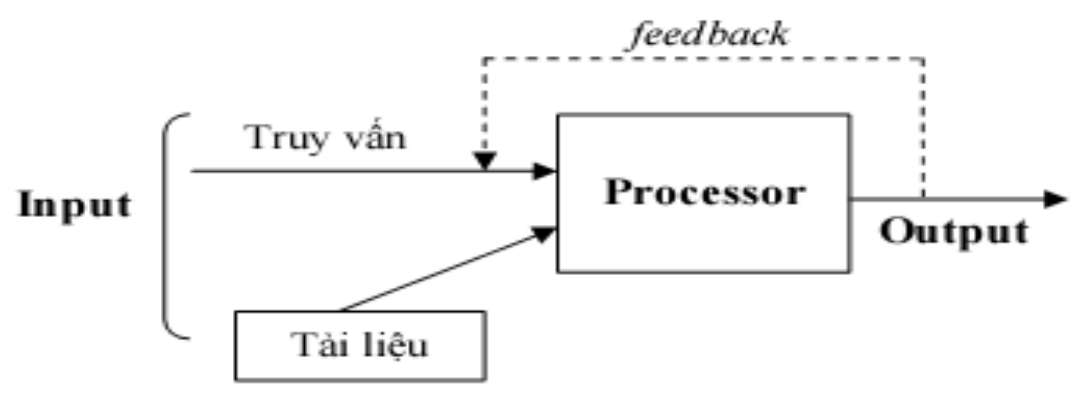

\section{Hình 1. Hệ thống IR}

Phần Processor: Là thành phần liên quan đến tiến trình truy tìm. Processor cần đến việc cấu trúc thông tin theo một số cách thích hợp như phân loại, hoặc có thể cần đến chiến lược tìm kiếm ứng với truy vấn.

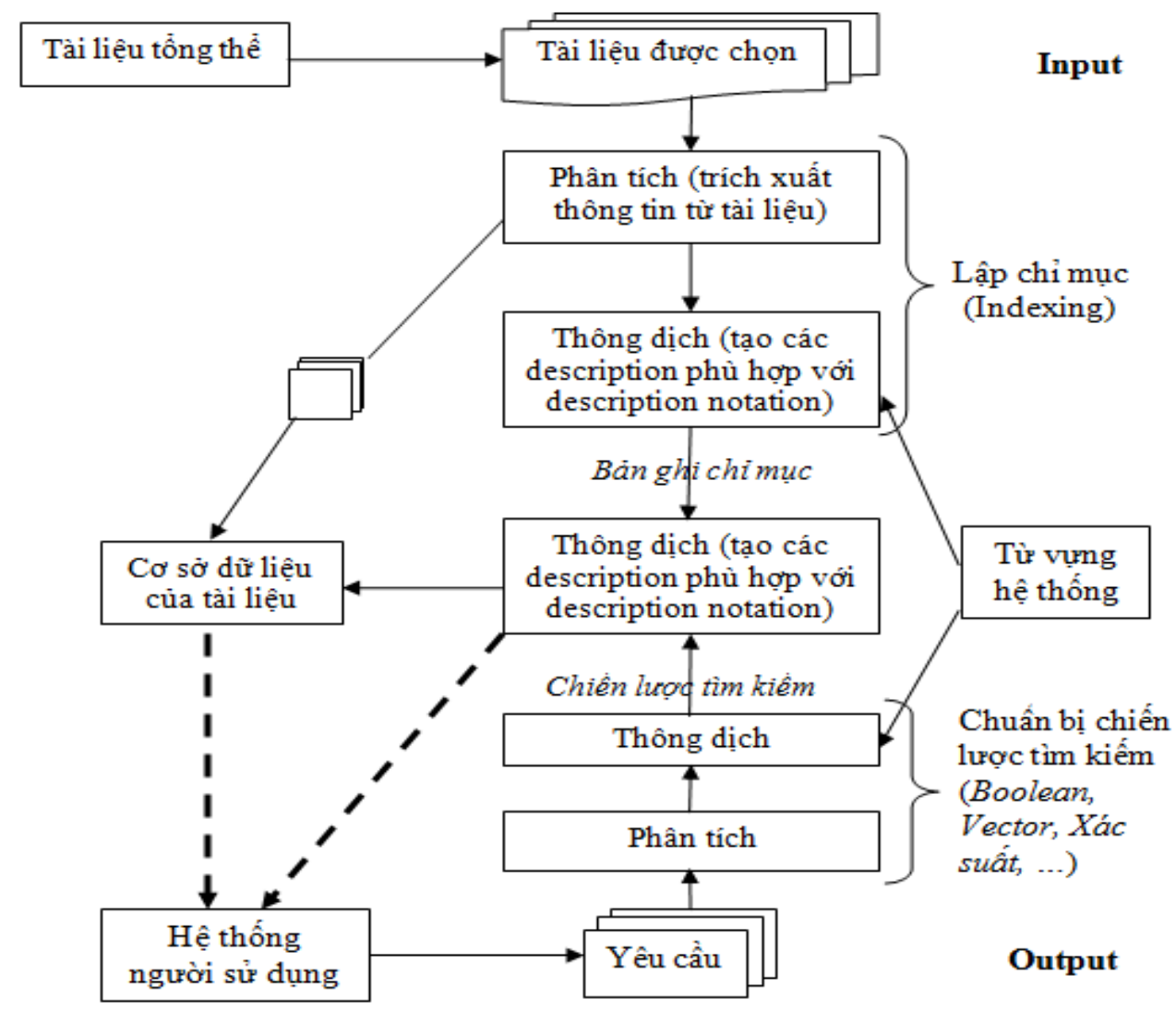

Hình 2. Các chức năng chính của hệ thống IR

Nguồn: Aly (2008). 
Phần Output: Là tập hợp trích dẫn hoặc các tài liệu được tìm thấy. Như thế có thể biết rằng một hệ thống IR không chỉ truy tìm thông tin mà còn nó còn truy tìm các tài liệu chứa thông tin nếu hệ thống đã đọc và hiểu được, vì thế ta xem đó IR là một hệ thống truy hồi thông tin.

\subsection{Các kỹ thuật cơ bản của IR}

Trong truy hồi thông tin có hai khái niệm luôn được đề cập đó là Document và Query. Document là bất kỳ đối tượng nào mà có chứa thông tin (ví dụ: các mẫu văn bản, hình ảnh, âm thanh, video, ...). Tuy nhiên hầu hết các hệ thống IR chỉ đề cập đến các document là tài liệu văn bản. Lý do về sự hạn chế này là vì cách thức thể hiện các đối tượng không phải là văn bản còn nhiều phức tạp. Query là sự thể hiện về thông tin mong muốn.

Thông tin được lưu bên trong máy tính thường ở dạng tài liệu (document). Tuy nhiên máy tính không thể lưu hết tất cả nội dung của tài liệu theo ngữ nghĩa tự nhiên mà tài liệu đó được viết. Thay vào đó, sẽ có một tài liệu đại diện được tạo ra bằng thủ công hoặc tự động, đây là quan điểm bắt nguồn cho tiến trình phân tích văn bản tự động. Cách thức thường dùng là thống kê ngôn ngữ để phục vụ cho việc phân tích văn bản tự động. Quan điểm ban đầu cho tiến trình phân tích văn bản được biểu diễn như Hình 3.

Toàn bộ nội dung văn bản (toàn văn)

Phần tóm tắt (abstract) của văn bản

Phần tiêu đề (title) của văn bản

Danh sách một vài từ
Các trường hợp này khi đó sẽ thành document tiêu biểu ở định dạng mà máy tính có thể xử lý được.

Hình 3. Quan điểm ban đầu cho tiến trình phân tích văn bản

\section{MÔ HÌNH VÀ GIẢI PHÁP QUẢN LÝ, TÌM KIẾM KHO TÀI LIẸU}

\subsection{Xây dựng mô hình lưu trữ ở mức quan niệm}

Để thực hiện mục tiêu tìm kiếm tài liệu theo mô hình thực tế, ta xây dựng mô hình kết hợp "Thu thập tài liệu, tổ chức lưu trữ, suy diễn trí thức" ở mức quan niệm như trong Hình 4. 


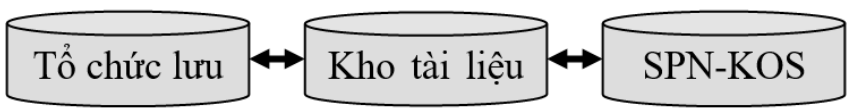

Hình 4. Mô hình ở mức quan niệm

Ghi chú: Software Programming Network - Keyphrase Ontology System (SPN-KOS)

Từ mô hình lưu trữ ở mức quan niệm, ta tiến hành phân tích hệ thống tài liệu và xây dựng mô hình tổng quát quản lý, lưu trữ tài liệu và tìm kiếm theo ngữ nghĩa từ “ Kho tài liệu thu thập được" đến "Mô hình cây thu mục quy chuẩn" cho đến "Mô hình quản lý theo ngũu nghĩa”. Trước tiên ta thu thập tài liệu về công nghệ thông tin tiếng Việt từ nhiều nguồn khác nhau, sau đó ta tổ chức lưu trữ theo cây thư mục quy chuẩn. Mỗi tài liệu sẽ được lưu trữ theo cây thư mục quy chuẩn lĩnh vực tương ứng với tài liệu đó. Cuối cùng dựa vào hệ trí thức SPN-KOS để suy diễn ngữ nghĩa cho từng tài liệu cụ thể.

\subsection{Tổ chức lưu trữ theo cây thư mục}

Các thư mục dùng để lưu trữ các tập tin được tổ chức theo mô hình SPN-KOS, gồm 66 thư mục chính, trong đó ta tạo mối quan hệ giữa các thư mục với nhau ở dạng liên kết như trong Hình 5. Ta xem mỗi thư mục tương ứng với một lớp trong SPN-KOS và cũng là một bucket trong bảng băm theo phương pháp nối kết trực tiếp, mỗi bucket chứa thông tin các trường gồm: Tên thư mục, ... Thư mục cha (parent). Trong đó trường Parent là một con trỏ sẽ trỏ đến một danh sách liên kết mà tại đó sẽ cho biết bucket hiện tại có nút cha là nút nào, và từ đó ta sẽ lấy được thư mục cha tương ứng.

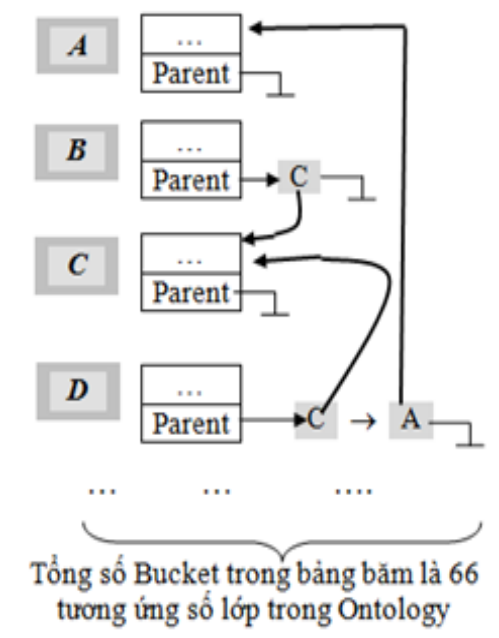

Hình 5. Tổ chức lưu trữ các thư mục 
Theo Hình 5 ta có bucket $\mathrm{D}$ có cha là $\mathrm{A}$ và $\mathrm{C}$, còn bucket $\mathrm{B}$ có cha là $\mathrm{C}$, đồng thời cũng biết rằng bucket $\mathrm{A}$ và $\mathrm{C}$ không có cha, như thế cũng biết được rằng bucket $\mathrm{A}$ và $\mathrm{C}$ chính là một trong số 3 lớp ban đầu của SPN-KOS (Hình 5). Để tổ chức lưu trữ, tìm kiếm theo ngữ nghĩa, từ mô hình mức quan niệm (Hình 4), ta xây dựng mô hình cơ sở dữ liệu phục vụ cho việc lưu trữ và tìm kiếm theo ngữ nghĩa.

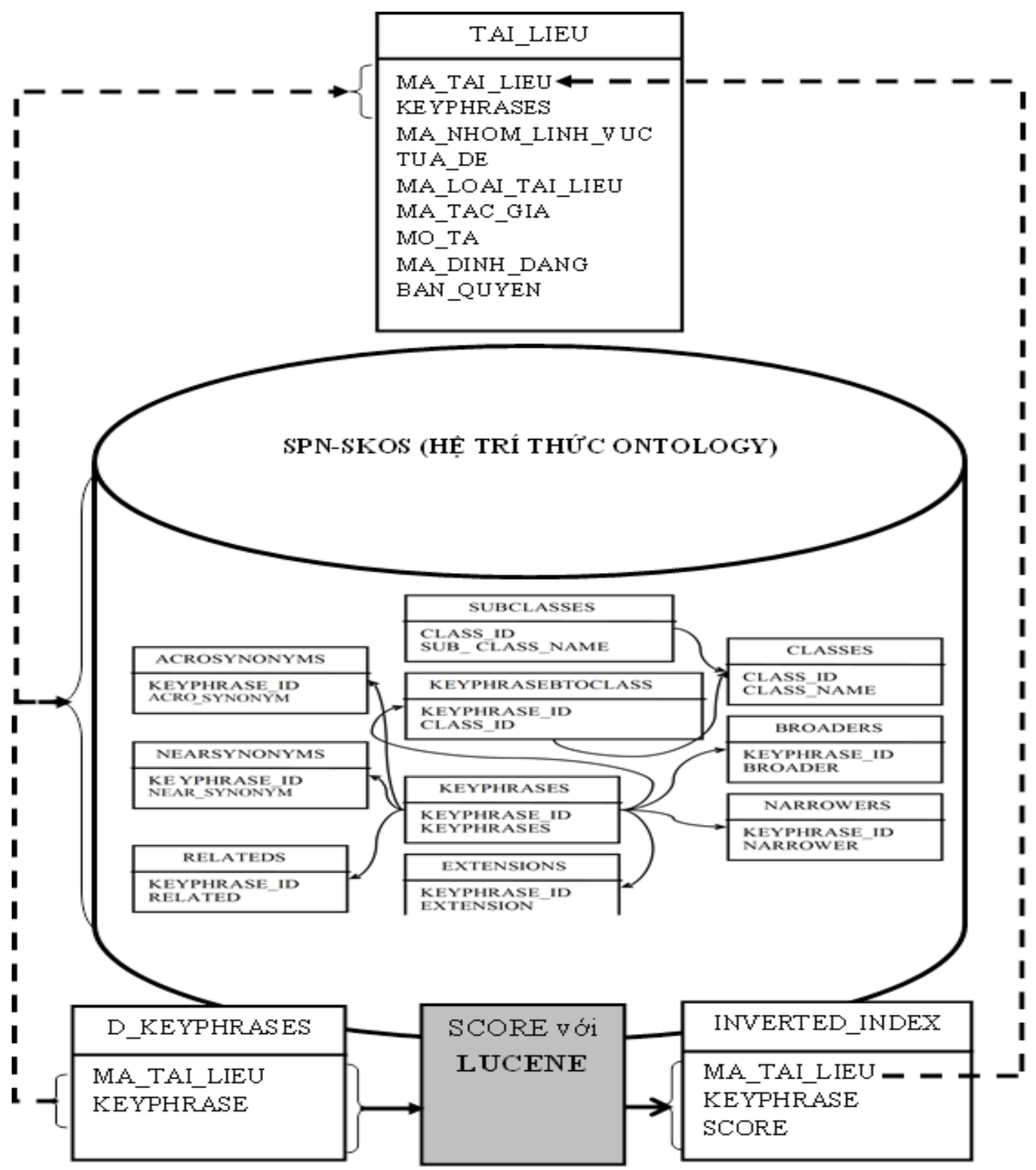

Hình 6. Mô hình cơ sở lưu trữ và tìm kiếm theo ngữ nghĩa 


\subsubsection{Thiết kế giải pháp và tổ chức kho tài liệu có ngũ nghĩa}

Ở giai đoạn này, quy trình thực hiện gồm năm bước như sau:

Buoớc 1. Rút trích keyphrases: Kết hợp với mô hình quản lý tài liệu theo cơ sở dữ liệu quan hệ, ta tiến hành rút trích các keyphrases của tài liệu bằng cách thủ công. Kết quả trả về là danh sách các keyphrases tương ứng với từng tài liệu cụ thể.

Bước 2. Phát sinh thêm các keyphrases: Kế tiếp dùng SPN-KOS để phát sinh thêm các keyphrases từ các mối quan hệ ngữ nghĩa (Acronym, Synonym, Near-Synonym, Broader, Narrower, Extension, Related) dựa trên các keyphrases đã có. Như thế thì số keyphrases gia tăng đều này cũng có thể khẳng định rằng tập tin mà ta đã trích ra các keyphrases được biểu diễn ngữ nghĩa nhiều hơn.

Bước 3. Tổ chức lưu trữ tập tin vào thư mục: Ngoài ra, ta cũng biết thêm rằng lớp chứa các keyphrases đó và khi cần xem lớp cha của lớp đó thì ta có thể biết thông qua cấu trúc tổ chức thư mục như trên. Cuối cùng, ta sẽ xét xem lớp nào mà số keyphrases bao hàm hầu hết các keyphrases của tập tin đang xét thì ta lưu tập tin đó vào thư mục tương ứng.

Ví dụ: Ta có tài liệu A gồm các keyphrases "C" và "hợp ngữ" sau đó ta dùng Ontology SPN-KOS để suy luận các mối quan hệ ngữ nghĩa có liên quan đến keyphrases này ta sẽ có thêm các keyphrases như sau: "ngôn ngữ máy”, "ngôn ngữ kết hợp”, "ngôn ngữ assembly", "KLOC”, “APL”, "C cộng cộng”, "ngôn ngữ lập trình", "C sharp”, "Eiffel”, "Ngôn ngữ cấp cao", "Ngôn ngữ mức cao", "Rexx”, "Visual C cộng cộng”. Có tổng cộng 15 keyphrases bao gồm các keyphrases bên trong tài liệu $\mathrm{A}$ và keyphrases suy diễn từ SPN-KOS (Bảng 1). Tổng số lớp liên quan với 15 keyphrases là 3 lớp: CÁC NGÔN NGỮ LẬP TRÌNH, CÔNG CỤ LẬP TRÌNH, LẬP TRÌNH. Trong đó lớp CÁC NGÔN NGƯ LẬP TRÌNH chứa 15 keyphrases trên, lớp LẬP TRÌNH chứa 1/15 keyphrases, lớp CÔNG CỤ LẬP TRÌNH chứa 1/15 keyphrases dẫn đến lớp CÁC NGÔN NGƯ LẬP TRÌNH chứa hầu hết các keyphrases vì thế ta lưu tập tin ban đầu (tập tin trước khi tiền xử lý) vào thư mục CÁC NGÔN NGỮ LẬP TRÌNH. 
Bảng 1. Các keyphrases trong lớp chứa tương ứng

\begin{tabular}{|c|c|}
\hline Keyphrases & Lớp chứa keyphrases \\
\hline $\mathrm{C}$ & CÁC NGÔN NGŨ LẬP TRÌNH \\
\hline hợp ngũ̃ & CÁC NGÔN NGỬ LẬP TRÌNH, CÔNG CỤ LẬP TRÌNH \\
\hline KLOC & CÁC NGÔN NGỪ LẬP TRÌNH, LẬP TRÌNH \\
\hline ngôn ngũ̃ assembly & CÁC NGÔN NGŨ̉ LẬP TRÌNH \\
\hline ngôn ngữ kết hợp & CÁC NGÔN NGŨ̉ LẬP TRÌNH \\
\hline ngôn ngữ máy & CÁC NGÔN NGỬ LẬP TRÌNH \\
\hline APL & CÁC NGÔN NGŨ̉ LẬP TRÌNH \\
\hline $\mathrm{C}$ cộng cộng & CÁC NGÔN NGŨ̉ LẬP TRÌNH \\
\hline C sharp & CÁC NGÔN NGŨ̀ LẬP TRÌNH \\
\hline Eiffel & CÁC NGÔN NGŨ̉ LẬP TRÌNH \\
\hline
\end{tabular}

Lưu đồ thuật toán lưu trữ tập tin vào thư mục được biểu diễn như trong Hình 7. Trong đó, đầu vào là tập tin dữ liệu ở dạng pdf, html, doc, txt; Đầu ra là tập tin đã được lưu vào trong thư mục tương ứng; Kết quả là các tài liệu được lưu vào thư mục tương ứng cùng keyphrases có quan hệ ngữ nghĩa.

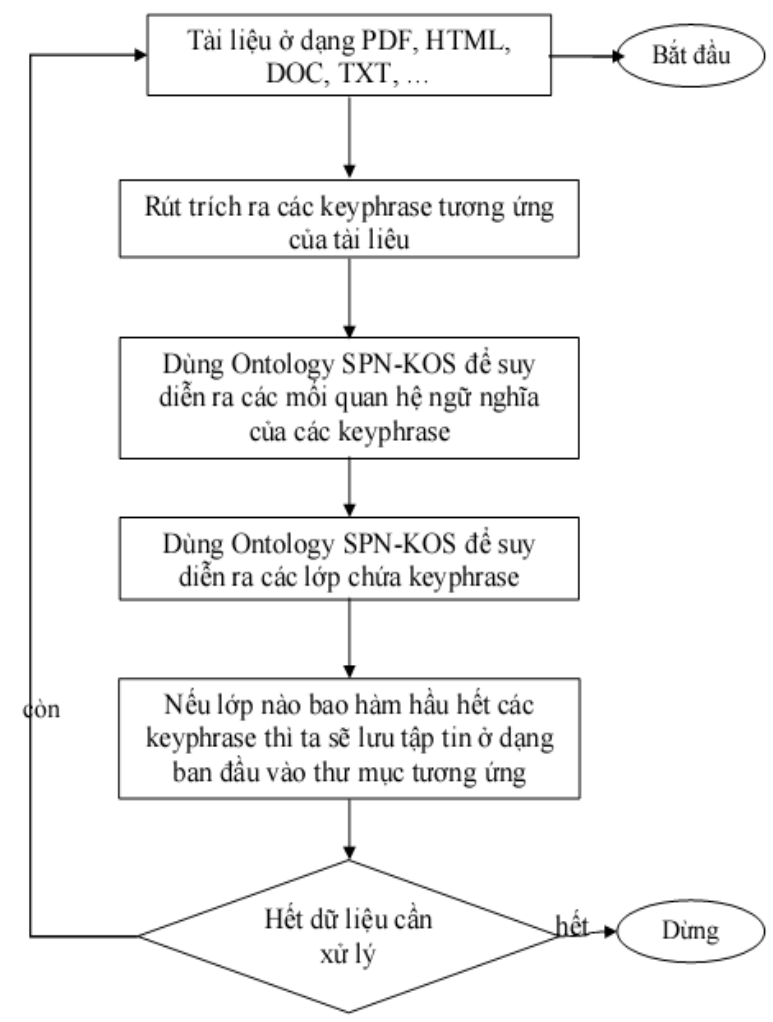

Hình 7. Lưu đồ rút trích và suy diễn ngữ nghĩa keyphrase 
Bước 4. Tính độ ưu tiên: Sau khi danh sách keyphrases được rút trích thủ công và suy diễn ngữ nghĩa cho từng keyphrase ở Bước 2 , ở bước này ta tính độ ưu tiên của keyphrases dựa vào Lucene. Trong đó đầu vào là danh sách các keyphrases; Đầu ra là mức độ ưu tiên cho từng keyphrases.

Bước 5. Xây dựng chỉ mục: Đầu vào là tập các keyphrases và các quan hệ Acronym, Synonym, Near-Synonym, Broader, Narrower, Extension, Related tương ứng với mỗi tài liệu; Đầu ra là danh sách các tập tin ứng với từng keyphrases cùng với mức độ ưu tiên. Các thành phần trong Indexing bao gồm: Dictionary: Lưu các danh sách keyphrases khác nhau đã được tạo suy diễn ngữ nghĩa trong từng tài liệu; DSTapTin: Danh sách mã tài liệu thứ i (MA_TAI_LIEU); và DSVitri: (keyphrases; DSTapTin): Tạo thành Indexing (Ontology trong lĩnh vực đang xét). Lưu đồ thuật toán được thể hiện như trong Hình 8.

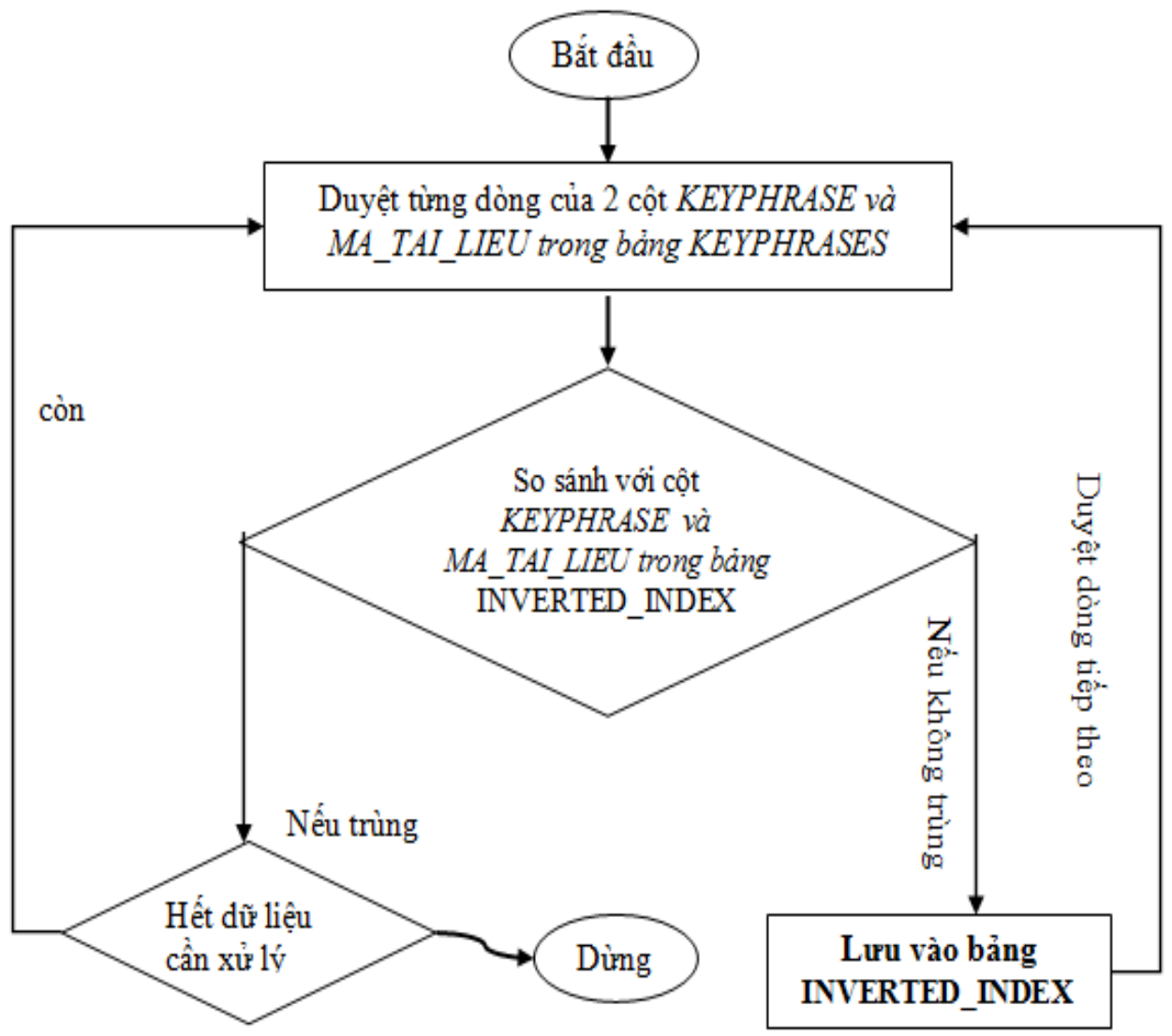

Hình 8. Lưu đồ lập chỉ mục cho tài liệu 


\subsubsection{Qui trình tìm kiếm theo ngũ nghĩa}

Ở giai đoạn này, quy trình cụ thể gồm ba bước như sau:

Bước 1. Xử lý rút trích keyphrases từ người dùng nhập vào: Ở giai đoạn này ta rút trích các keyphrases có nghĩa do người dùng nhập vào (kết quả danh sách keyphrases chưa có quan hệ) dựa vào kho tự điển. Lưu đồ thuật toán thể hiện như trong Hình 9 . Trong đó đầu vào là câu truy vấn do người dùng nhập vào; Đầu ra là danh sách các keyphrases rút trích được từ người dùng. Ví dụ, người dùng nhập vào câu truy vấn "Giao thức truyền tập tin" thì kết quả rút trích keyphrases có nghĩa sau:

Danh sách keyphrases có nghĩa = [Giao thức, Giao thức truyền tập tin, tập tin]

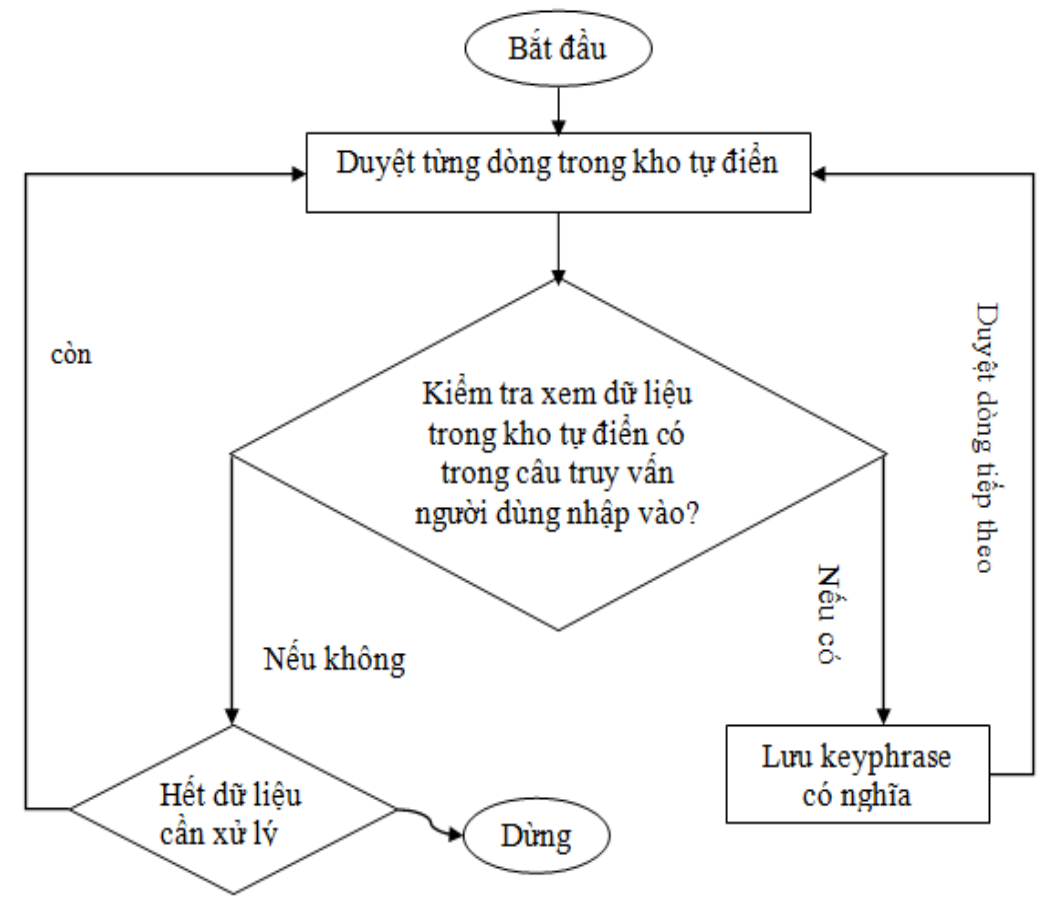

Hình 9. Lưu đồ rút trích keyphrases từ câu truy vấn của người sử dụng

Bước 2. Suy diễn ngữ nghĩa: Trong bước này, đầu vào là các keyphrases được rút trích ở Bước 1; Đầu ra là các keyphrases có quan hệ ngữ nghĩa (Acronym, Synonym, Synonym, Broader, Narrower, Extension, Related).

Bước 3. Tìm kiếm văn bản có chứa các keyphrases: Từ danh sách kết quả do người dùng nhập vào và được biểu diễn ngữ nghĩa ở Bước 2 và dựa vào INVERTED_INDEX 
(Kết quả ở Bước 5 mục 4.2.1), ta tiến hành tìm kiếm nội dung tài liệu có chứa các keyphrases do người dùng nhập vào và xếp hạng keyphrases có độ ưu tiên lớn nhất trả về. Kết quả trả về là danh sách các tài liệu có chứa các keyphrases do người dùng nhập vào.

\subsection{Mô hình tổ chức lưu trữ tổng thể, tìm kiếm tài liệu theo ngữ nghĩa}

Mô hình tổng thể quản lý, tìm kiếm hệ thống tài liệu theo hướng có ngữ nghĩa được thực hiện hai giai đoạn đó là: (i) Xây dựng tài liệu theo hướng có ngữ nghĩa; và (ii) Xử lý tìm kiếm theo ngữ nghĩa. Lưu đồ lưu trữ tài liệu theo hướng ngữ nghĩa được biểu diễn như trong Hình 10.

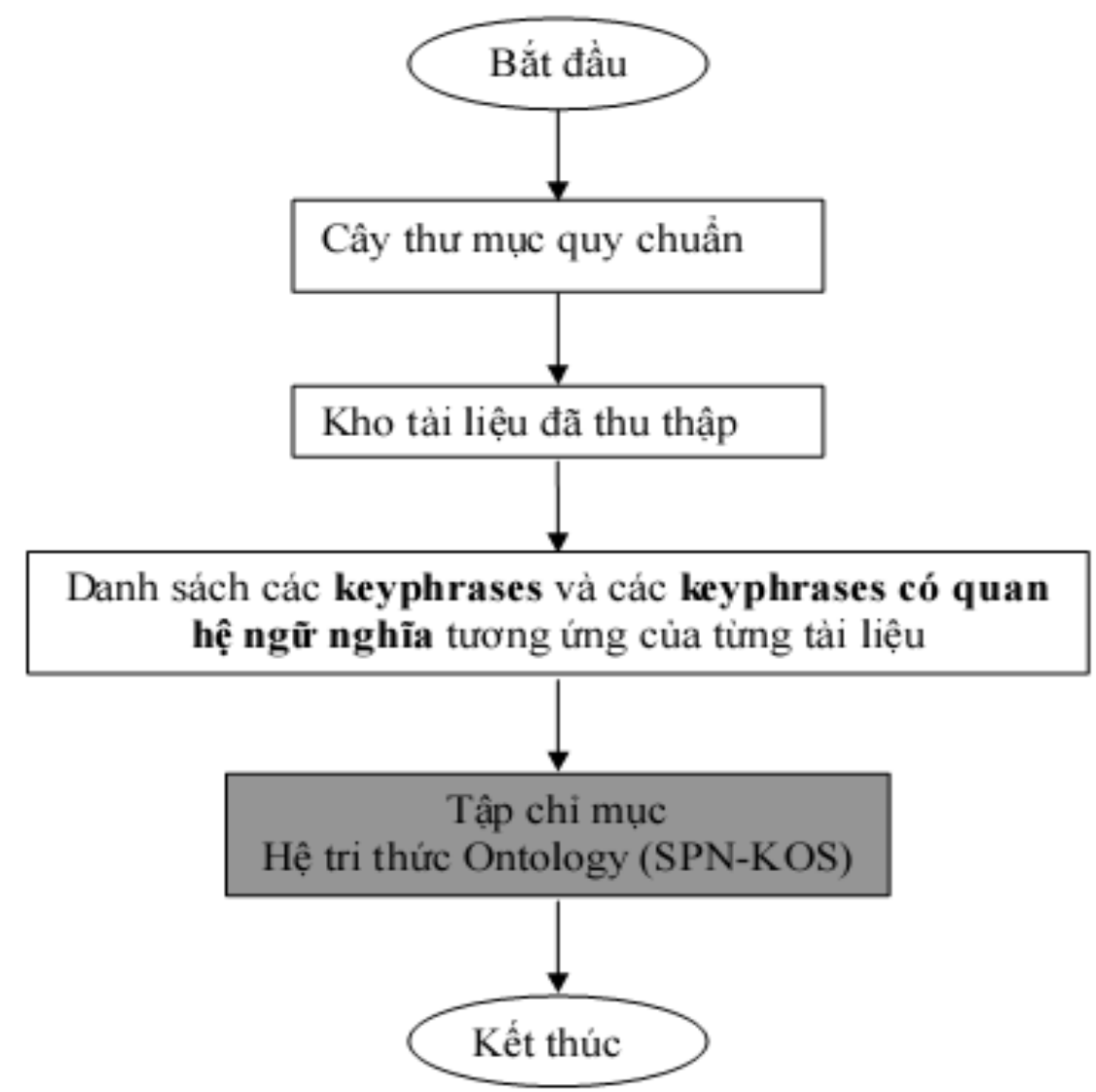

Hình 10. Mô hình quản lý tài liệu 


\subsection{Lưu đồ xử lý tìm kiếm tài liệu theo hướng có ngữ nghĩa}

Lưu đồ tìm kiếm theo ngữ nghĩa được trình bày trong Hình 11. Trong lưu đồ này, đầu vào là câu người dùng nhập vào truy vấn; Đầu ra là danh sách các tài liệu liên quan đến nhu cầu tìm kiếm của người dùng được sắp xếp theo độ ưu tiên.

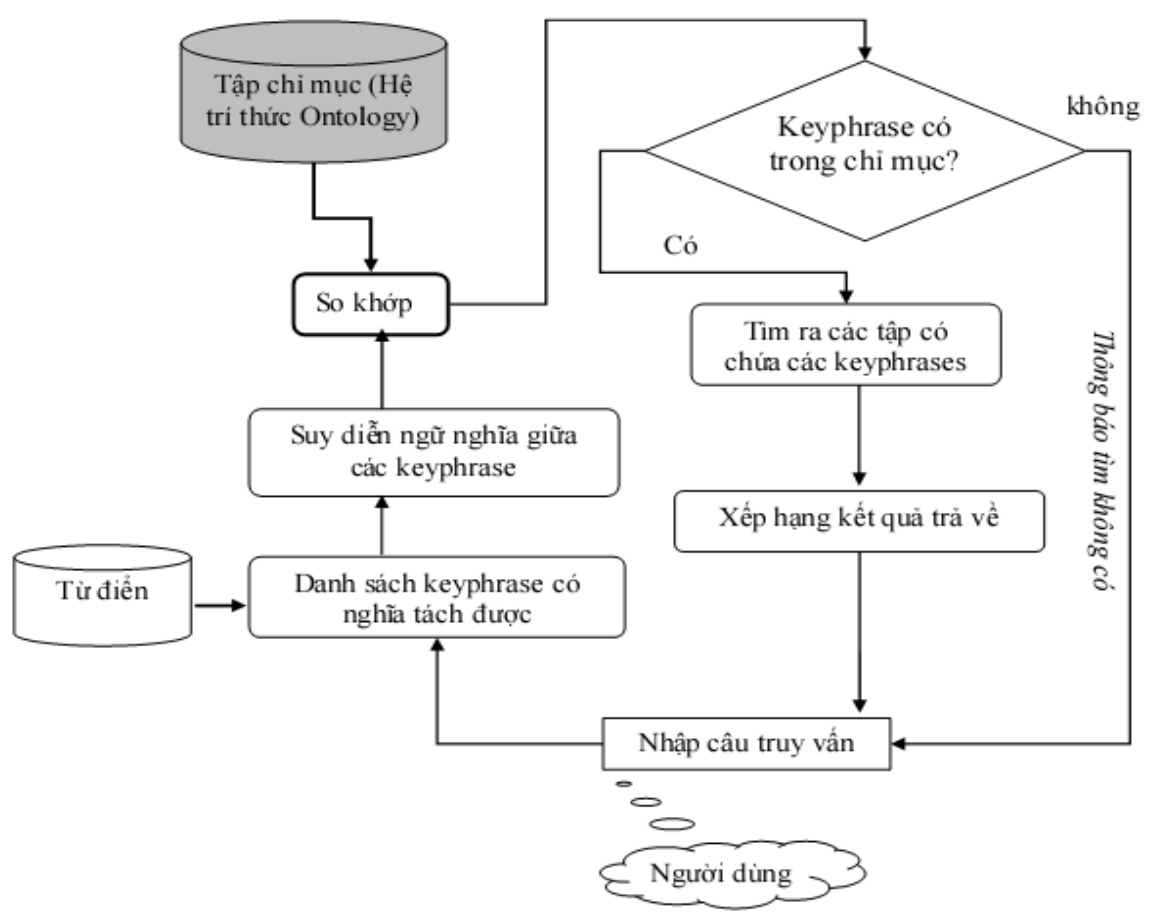

Hình 11. Lưu đồ tìm kiếm theo ngữ nghĩa

\section{THỰC NGHIỆM VÀ KẾT QUẢ}

\subsection{Giới thiệu về chương trình}

Dựa vào mô hình và giải pháp nêu ở Mục 4, ta xây dựng chương trình ứng dụng thử nghiệm. Trong chương trình ứng dụng này có 3 phân hệ chính:

- Quản trị tài liệu: Người sử dụng có thể thêm/xóa/sửa tài liệu khi có nhu cầu.

- Suy diễn quan hệ ngữ nghĩa: Ở phân hệ này người sử dụng suy diễn mối quan hệ ngữ nghĩa cho các tài liệu dựa vào các keyphrases được trích thủ công.

- Tìm kiếm: Phân hệ tìm kiếm giúp người sử dụng tìm kiếm tài liệu theo ngữ nghĩa. Khi người sử dụng nhập vào một câu tìm kiếm, phân hệ này phân tích 
các keyphrases có nghĩa và suy diễn mối quan hệ ngữ nghĩa giữa các keyphrases, tìm kiếm các tài liệu có keyphrases tương ứng và trả về kết quả theo thứ tự giảm dần.

Cuối cùng, ta tiến hành đo hiệu suất thu thập thông tin của chương trình tìm kiếm theo ngữ nghĩa trên các mẫu ví dụ thông qua độ recall, precision kết hợp với hệ số. Để tiến hành đánh giá kết quả thử nghiệm cho giải pháp tìm kiếm theo ngữ nghĩa, ta thử nghiệm trên hai mẫu ví dụ sau: Ví dụ Q1: Tìm kiếm tài liệu có liên quan đến "Giáo trình lập trình C"; Ví dụ Q2: Tìm kiếm tài liệu có liên quan đến "Giao thức truyền tập tin".

\subsection{Xây dụng hệ thống}

Ở giai đoạn này ta tiến hành phân tích khảo sát nghiệp vụ chuyên môn và thực hiện một số công đoạn thủ công.

Thu thập kho tài liệu: Việc thu thập và phân loại kho dữ liệu bằng thủ công ta được hơn $6 \mathrm{~GB}$ về 3 lĩnh vực: Phần mềm, Lập trình, Mạng máy tính từ nhiều nguồn khác nhau để tiến hành thử nghiệm chương trình.

Tạo cấu trúc cây thư mục: Trong Mục 4.2 có đề xuất các thức tạo cấu trúc thư mục để lưu trữ tập tin tương ứng với bảng băm gồm các bucket (là thư mục) và danh sách liên kết đi kèm để biết thư mục cha là gì. Mỗi thư mục là sự tương ứng với lớp trong Ontology mỗi thư mục sẽ chứa các tập tin mà mỗi tập tin đó có các keyphrases hầu hết được bao hàm bởi thư mục chứa nó.

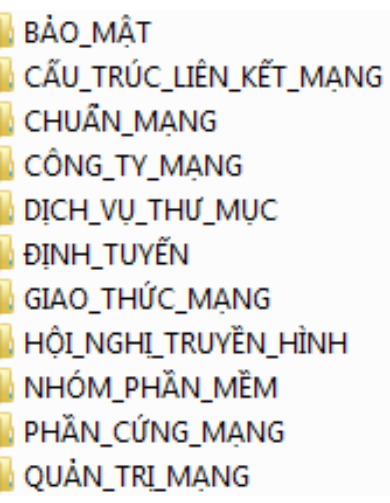

Hình 12. So đồ lưu trữ mức vật lý cấu trúc thư mục 
Xây dụng từ điển keyphrases: Việc phân loại lớp trong chuyên ngành đòi hỏi cần có chuyên gia về lĩnh vực đó. Vì vậy việc phân loại lớp bằng thủ công dựa vào các trang web www.dmoz.org/Computers/; dir.yahoo.com/Computers_and_Internet/; và trang web về tự điển máy tính www.webopedia.com. Từ đó ta được 66 lớp ở dạng chưa phân cấp như trong Hình 13.

\begin{tabular}{|c|}
\hline inClass \\
\hline BẢNG TÍNH \\
\hline BẢO MÂTT \\
\hline BIÊN DİCH-ĐÓNG GÓI-LIÊN KÉT \\
\hline BIÊU THƯC \\
\hline CÁC CÂU TRUY VÀN \\
\hline CÁC CHUÃN ĐIING DANG \\
\hline CÁC CHUÂN ĐİNH DANG \\
\hline CÁC CÔNG TY MANG \\
\hline CÁC GIAO DIÊN LÂP TRİNH ƯNG DỤNG \\
\hline CÁC LO̊I ÚNG DỤNG \\
\hline CÁC LỚP CƠ SỚ \\
\hline CÁC NGÔN NGỮ LÂP TRINH \\
\hline CÁC TIÊU CHUÂN MANGG \\
\hline
\end{tabular}

\section{Hình 13. Các lớp ở dạng chưa phân cấp}

Từ 66 lớp chưa phân cấp ta sẽ xây dựng cây phân cấp thư mục theo các hai bước:

Bước 1. Ở giai đoạn này ta xây dựng 3 lớp chính cho SPN-KOS như sau:

$P(E)$ có các tập con là: $\mathrm{C}_{1}=$ "PHẦN MỀ", $\mathrm{C}_{2}=$ "LẬP TRİNH", $\mathrm{C}_{3}=$ "MẠNG MÁY TÍNH”. Ta xây dựng cây phân cấp cho SPN-KOS như trong Hình 14.

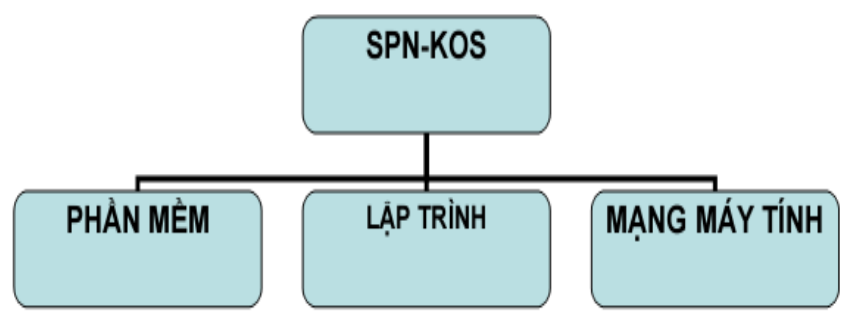

Hình 14. Tổng quan về SPN-KOS và các lớp của nó

Như vậy cơ bản sẽ có 3 lớp (tập hợp) chứa trong $P(\mathrm{E})$ như sau:

$$
\forall \mathrm{i}=1,2,3: \mathrm{C}_{\mathrm{i}} \subseteq E \text {, và } P(\mathrm{E}) \supset\left\{\mathrm{C}_{1}, \mathrm{C}_{2}, \mathrm{C}_{3}\right\}
$$


Bước 2 . Từ 3 lớp phân cấp ban đầu ta tiếp tục xây dựng 44 lớp tiếp theo dựa vào dữ liệu cha-con, Sau đó ta lại tiếp tục xây dựng được 19 lớp mới vì nó là cấp con của 44 lớp vừa xây dựng.

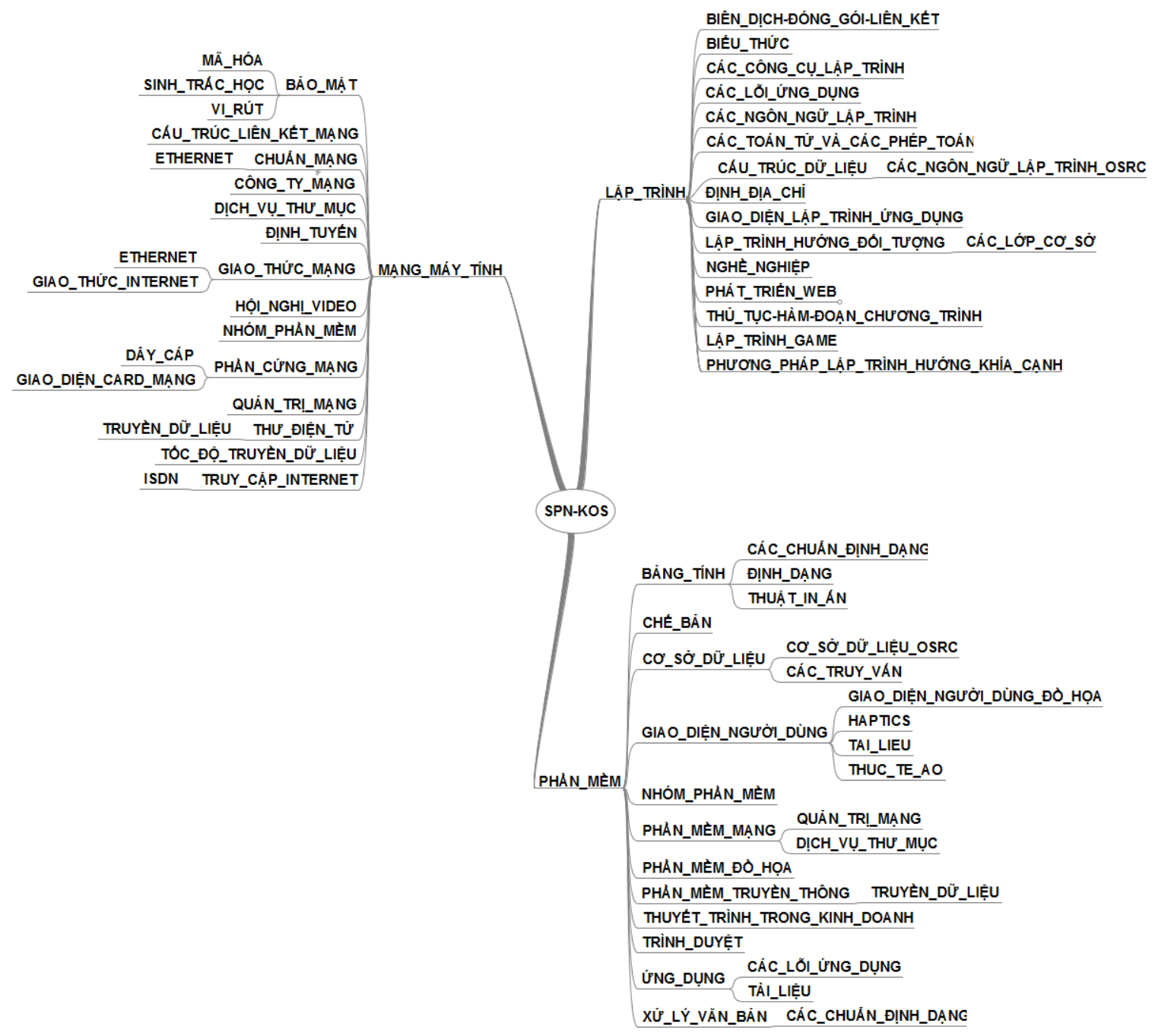

Hình 15. Mô hình cây phân cấp SPN-KOS

Ví dụ về cách xây dựng lớp LẬP TRÌNH và các lớp liên quan như sau: Lớp này có 20 lớp con: (LẬP TRÌNH GAME, LẬP TRİNH HƯƠNG ĐỐI TƯợNG, BIÊN DỊCHĐÓNG GÓI VÀ LIÊN KÊT, ELECTRONIC TECHNOLOGY, BIẾU THỨC, CÁC NGÔN NGỮ LẬP TRÌNH, CÁC TOÁN TƯ VÀ CÁC PHÉP TOÁN, CÂU TRÚC DŨ LIỆU, ĐỊNH ĐỊA CHİ, GIAO DIỆN LẬP TRİNH ỨNG DỤNG, NGHỀ NGHIỆP, PHÁT TRIỄN WEB, PHƯONG PHÁP LẬP TRÌNH HƯỚNG KHÍA CẠNH, THỦ 
TỤC-HÀM-ĐOẠN CHƯƠNG TRÌNH, CÁC NGÔN NGỬ LẬP TRÌNH OSRC, HTML, XML, TRİNH DUYẸT, CÁC LỚP CƠ SỞ). Như vậy sẽ có 20 lớp con sẽ được tạo, nếu lớp nào trùng với lớp đã có thì không tạo nữa. Cho nên ta xét tiếp các lớp con của lớp LẬP TRÌNH như sau: Trong 20 lớp con của LẬP TRÌNH, thì có một số lớp đồng thời vừa là lớp con của LẬP TRÌNH và cũng là lớp con của lớp khác.

XML $\subset$ LẬP TRİNH, PHÁT TRIỂN WEB. Từ đó lớp XML vừa là con của LẬP TRÌNH và là con của PHÁT TRIỂN WEB.

TRİNH DUYỆT $\subset$ HTML, PHẦN MỀM nên lớp TRÌNH DUYẸT vừa là con của HTML và PHẦ MỀM.

Như thế trong quá trình xét duyệt 3 lớp cơ bản ta tạo được thêm 20 lớp. Tiếp tục xét các lớp con của 44 lớp và tạo tiếp 19 lớp cũng theo ý tưởng tương tự như sau:

CHUẨN MẠNG つ ETHERNET nên lớp CHUÂNN MẠNG có lớp con là ETHERNET nhưng lớp con này đã được tạo trong số 19 lớp nên không tạo nữa.

CƠ SỞ DŨ̉ LIẸU つ (CƠ SỞ DỬ LIỆU OSRC, CÁC CÂU TRUY VẤN ) nên lớp CƠ SỞ DŨ LIỆ có lớp con là OSRC CƠ SỞ DŨ LIỆU OSRC, CÁC CÂU TRUY VẤN trong đó lớp OSRC DATABASES đã được tạo nên không tạo, còn lớp CÁC CÂU TRUY VÂN chưa có mặt trong số 41 lớp cho nên tạo lớp này.

HAPTICS つ GIAO DIỆN NGƯỜI DÙNG nên lớp HAPTICS có lớp con là GIAO DIỆN NGUOÒI DƯNG và lớp này không có trong 169 nên tạo thêm lớp này.

GIAO THƯC INTERNET $\supset$ GIAO THƯCC MẠNG nên lớp GIAO THƯC MẠNG có lớp con là GIAO THỨC INTERNET và lớp này chưa được tạo trong số 19 lớp cho nên ta cần tạo thêm.

Mỗi lớp được hình thành có chứa tập các keyphrases để mô hình cho một phạm vi cụ thể. So với tập cha của nó. Như thế ta có tập $C=\bigcup_{i=1}^{66} C_{i}$ sẽ gồm 66 lớp là tổng số lớp theo thống kê trên, mỗi lớp sẽ chứa một số keyphrase trong $E$, ký hiệu $C_{i}=\left\{i_{1}, i_{2}, \ldots i_{n}\right\}$, và 
theo thống kê ta có được 76 mối quan hệ is-a giữa 66 lớp. Và ta có $E=\bigcup_{i=1}^{n} C_{i}$ với $\mathrm{n}=66$ lớp.

Quan hệ giữa các keyphrases trong E: Ta có tập $E \neq \varnothing$, một quan hệ 2 ngôi trên $E$ là một tập hợp con $R$ của $E^{2}$. Cho 2 phần tử x và y của $E$, ta nói x có quan hệ $\mathrm{R}$ với $\mathrm{y}$ khi và chỉ khi $(x, y) \in R$, và viết là $x R y$. Như vậy: $x \mathrm{Ry} \Leftrightarrow(\mathrm{x}, \mathrm{y}) \in \mathrm{R}$. Khi x không có quan hệ $\mathrm{R}$ với $\mathrm{y}$, ta viết: $x \bar{R} y$. Các quan hệ giữa các keyphrases trong $\mathrm{E}$ bao gồm: Quan hệ cùng lớp giữa các keyphrases: Ta nói keyphrase $a$ có quan hệ cùng lớp $\left(\mathrm{r}_{1}\right)$ với keyphrase $b$ nếu có một $\mathrm{C}_{\mathrm{i}}$ sao cho $\mathrm{a} \in \mathrm{C}_{\mathrm{i}}$ và $\mathrm{b} \in \mathrm{C}_{\mathrm{i}}$ (Hình 16); Quan hệ broader - narrower giữa các keyphrases: Ta nói keyphrase $a$ có quan hệ broader $\left(\mathrm{r}_{2}\right)$ với keyphrase $b$ nếu $a$ có nghĩa bao hàm $b$, hoặc có thể hiểu $a$ có nghĩa rộng hơn so với $b$ trong một ngữ cảnh đang xét và ngược lại ta nói $\mathrm{b}$ có quan hệ narrower $\left(r_{2}^{-1}\right)$ so với $\mathrm{a}$. Về ngữ cảnh như thế nào thì cần dựa theo định nghĩa được cho tại Webopedia để phân tích và thiết lập.

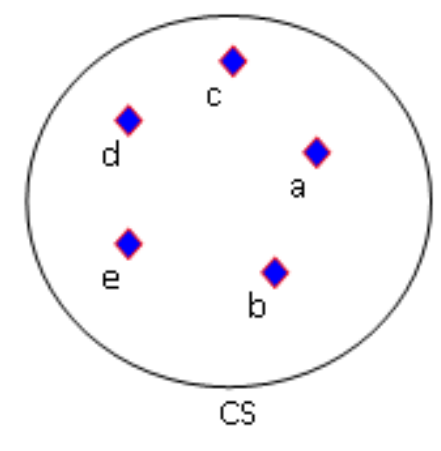

\section{Hình 16. Quan hệ cùng lớp giữa các keyphrases}

Xét định nghĩa của keyphrases AppleTalk Address Resolution Protocol như sau: "Short for AppleTalk Address Resolution Protocol, a protocol for mapping a device's physical hardware address to a temporary Appletalk network-assigned address in Macintosh computer LANs. When a protocol stack sends a data packet, the protocol address specifies the destination. The data link layer relies on AARP to translate the protocol address into the hardware address of the destination node". Như thế trong quá trình đọc và xét duyệt thủ công ta có thể tạo nên mối quan hệ broader - narrower giữa các keyphrases. Theo Hình 17, có thể hiểu các keyphrases ở cột KeyphraseName là narrower 
so với keyphrase "giao thức". Mối quan hệ is-a giữa các lớp có thể mô tả thành mối quan hệ broader - narrower giữa các keyphrases nếu keyphrases đó có tên giống với tên lớp và nó được chứa trong lớp đó.

\begin{tabular}{l|l}
\multicolumn{1}{c|}{ KeyphraseName } & \multicolumn{1}{c}{ Broader } \\
\hline Giao thức chèn AppleTalk & giao thức \\
\hline phương thức truyền tập tin & giao thức \\
\hline Giao thức cổng miền trong & giao thức \\
\hline phương thức truyền tệp & giao thức \\
\hline Ethernet & giao thức \\
\hline phương thức định tuyến & giao thức \\
\hline giao thức xác thực mớ rộng bảo vệ & giao thức \\
\hline Giao thức truyền siêuu văn bản & giao thức \\
\hline Mô hình Phân bố thành phần đối tượng & giao thức \\
\hline goị thủ tục tứ xa & giao thức \\
\hline phương thức quảng cáo dịch vụ & giao thức \\
\hline Phương pháp gọi từ xa & giao thức \\
\hline
\end{tabular}

\section{Hình 17. Keyphrases có broader là keyphrases "giao thức"}

Ta có mối quan hệ giữa lớp NGÔN NGŨ LẬP, đồng thời ta cũng có keyphrase ngôn ngũu lập trình (programming language) $\in$ NGÔN NGŨ LẬP TRİNH. Như vậy keyphrases có tên giống tên lớp sẽ mối quan hệ broader - narrower giữa chúng. Đồng thời cần đọc thêm định nghĩa bằng ngôn ngữ tự nhiên để xác định thêm keyphrases ngôn ngũu lập trình có mối quan hệ narrower với các keyphrases là $B A S I C, C, C++, C O B O L$, FORTRAN, Ada, Pascal dựa theo định nghĩa của ngũ lập trình được cho tại Webopedia như sau: "A vocabulary and set of grammatical rules for instructing a computer to perform specific tasks. The term programming language usually refers to high-level languages, such as BASIC, C, C++, COBOL, FORTRAN, Ada, and Pascal. Each language is a unique set of keywords (words that it understands) and a special syntax for organizing program instructions .....”. Như vậy mối quan hệ broader - narrower của các keyphrases có được do xuất phát từ mối quan hệ giữa các lớp và ta ký hiệu như sau:

$$
\begin{aligned}
& \text { a broader } \mathrm{b} \Leftrightarrow \operatorname{class}(\mathrm{a}) \supset \operatorname{class}(\mathrm{b}) \\
& \text { a narrower } \mathrm{b} \Leftrightarrow \operatorname{class}(\mathrm{a}) \subset \operatorname{class}(\mathrm{b})
\end{aligned}
$$


Trong đó: class(a) là lớp chứa keyphrases a; class(b) là lớp chứa keyphrases b. Quy ước class(x) là lớp chứa keyphrases $\mathrm{x}$.

Quan hệ đồng nghĩa (synonym) giữa các keyphrases: Ta nói keyphrases $a$ có quan hệ đồng nghĩa hoặc ở dạng rút gọn $\left(\mathrm{r}_{3}\right)$ với keyphrases $b$ nếu trong một ngữ cảnh đang xét ta đều có hai keyphrases cùng ý nghĩa. Ví dụ xét định nghĩa của keyphrases packet được cho như sau: "A piece of a message transmitted over a packet-switching network. See under packet switching. One of the key features of a packet is that it contains the destination address in addition to the data. In IP networks, packets are often called datagrams". Như vậy keyphrases packet có quan hệ đồng nghĩa $\mathrm{r}_{3}$ với keyphrases datagram trong ngữ cảnh computer network.

Quan hệ gần nghĩa (near-synonym) giữa các keyphrases: Ta nói keyphrases $a$ có gần nghĩa $\left(\mathrm{r}_{4}\right)$ với keyphrases $b$ nếu trong một ngữ cảnh đang xét ta đều có hai keyphrases có cùng ý nghĩa, hoặc ý nghĩa của chúng gần giống nhau. Ví dụ xét định nghĩa của keyphrases mã truy cập (access code) trong đó có câu định nghĩa: "Same as password, a series of characters and numbers that enables a user to access a computer". Như vậy keyphrases mã truy cập (access code) có keyphrases gần nghĩa là mật khẩu (password). Hay xét định nghĩa của keyphrases ActiveX: "ActiveX is a loosely defined set of technologies developed by Microsoft in 1996 for sharing information among different applications. ActiveX is an outgrowth of two other Microsoft technologies called OLE (Object Linking and Embedding) and COM (Component Object Model). As a moniker, ActiveX can be very confusing because it applies to a whole set of COM-based technologies. Most people, however, think only of ActiveX controls, which represent a specific way of implementing ActiveX technologies. Many Microsoft Windows applications use ActiveX controls". Như vậy keyphrases ActiveX có keyphrases gần nghĩa là COM và keyphrase OLE.

Quan hệ extension giữa các keyphrasess: Ta nói keyphrase $a$ có quan hệ extension $\left(\mathrm{r}_{5}\right)$ với keyphrases $b$ nếu keyphrase $a$ có chứa các ý nghĩa như $b$ và $a$ còn có thêm thông bổ sung cho nó, hoặc nghĩa của keyphrase a nâng cấp từ keyphrase b, hoặc nghĩa của 
keyphrase a thay thế ý nghĩa của keyphrase b, hoặc keyphrase sa là dẫn xuất từ keyphrase b và ý nghĩa bổ sung ngữ cảnh, hoặc keyphrase a là dựa trên keyphrase b. Để thiết lập được mối quan hệ này thì cần dựa theo các định nghĩa bằng ngôn ngữ tự nhiên được cung cấp từ Webopedia. Ví dụ xét định nghĩa về $\mathrm{C}++$ được cho tại Webopedia như sau: "A highlevel programming language developed by Bjarne Stroustrup at Bell Labs. C++ adds object-oriented features to its predecessor, C. C++ is one of the most popular programming language for graphical applications, such as those that run in Windows and Macintosh environments."

Dựa theo một phần của định nghĩa đã cho ta có thể thiết lập giữa keyphrases $C$ và keyphrases $\mathrm{C}++$ đó là $\mathrm{C}++$ là extension của $\mathrm{C}$. Sự có liên hệ giữa các keyphrases: Ta nói keyphrases a có liên hệ related ( $\mathrm{r}_{6}$ ) với keyphrases $\mathrm{b}$ nếu đó được liệt kê tại web. Sự có liên hệ này mang tính phỗ quát mà nó có thể bao hàm các mối quan hệ đã đề cập trên, hoặc nó vừa có ý nghĩa trong quan hệ này và có ý nghĩa trong quan hệ khác, do đó Webopedia cũng đã cung cấp sẵn các keyphrases có liên hệ với keyphrases mà ta đang xét. Một ví dụ khác, xét keyphrases "database” có các keyphrases liên quan được liệt kê cụ thể tại Webopedia như sau: "Active archiving, attribute, connection pool, DAM, data mining, data warehouse, database management system, distributed database, DML, drill down, DSN, dynaset, EDGAR, ETL, field, file, hypertext, metadata, ODS, OLAP, RDBMS, record, replication".

Để xác định các mối quan hệ $r_{2}, r_{3}, r_{4}, r_{5}$ ta đều dựa theo định nghĩa bằng ngôn ngữ tự nhiên được cung cấp tại Webopedia, trong quá trình tạo thủ công cần xem xét các định nghĩa liên quan với một keyphrases chứ không phải chỉ xét trên trong phạm vi định nghĩa của keyphrases được cho. Còn mối quan hệ $\mathrm{r}_{6}$ được Webopedia liên kết kèm theo các định nghĩa. Sau đây ta xét thêm một vài định nghĩa và phân tích nó:

Định nghĩa của analog television: "Preceding digital television (DTV), all televisions encoded pictures as an analog signal by varying signal voltage and radio frequencies. DTV is fast replacing analog TVs as digital broadcasting enables broadcasters to offer television with movie-quality picture and sound. Analog systems are more commonly known as NTSC systems. A U.S. Senate panel is set an April 7, 2009, as the 
deadline for television stations to switch entirely from analog to digital broadcasts. Analog televisions will work until all analog broadcasting ceases. Once the transition to complete $D T V$ is taken place, a converter will be required to receive DTV signals and change them to the analog format of these older types of televisions. However, these DTV-to-analog converters will not produce true DTV quality. Analog televisions are now commonly referred to conventional televisions."

Trong định nghĩa của analog television có ghi như sau "Preceding digital television". Điều này cho biết analog television có extension của nó là digital television hay có thể nói rằng digital television là extendFrom analog television khi đó ta có thêm mối quan hệ extension giữa analog television và digital television. Ngoài ra, phần cuối của định nghĩa analog television có để cập "Analog televisions are now commonly referred to conventional televisions". Từ đây ta cũng có thể suy luận rằng analog television đồng nghĩa với conventional televion như vậy ta có thể mối quan hệ synonym giữa analog television với conventional televion.

Xét định nghĩa về crimeware như sau: "A type of malicious software that is designed to commit crimes on the Internet. Crimeware may be a virus, spyware or other deceptive piece of software that can be used to commit identity theft and fraud". Theo định nghĩa và phân tích ta nhận thấy crimeware có broader là malicious software, crimeware có near-synonyms là virus, spyware.

Xét định nghĩa DAML như sau: "Short for DARPA Agent Markup Language, $D A M L$ is a semantic markup language that is specifically an extension to XML and the Resource Description Framework (RDF). DAML is used for the U.S. Defense Advanced Research Project Agency (DARPA) and compared to the XML standard it offers a better capacity to express semantics (describing objects and the relationships between objects), which means a much higher level of interoperability between Web sites". Ta có broader của DAML là semantic markup language và $\mathrm{DAML}$ extension từ $X M L$ và $R D F$. Giữa quan hệ broader và quan hệ near-synonym tùy thuộc vào ngữ cảnh và định nghĩa được cho mà 
nó sẽ có những trường hợp broader và near-synonym giống nhau và cũng có trường hợp là khác nhau.

Xét định nghĩa của keyphrase "multidimensional DBMS" như sau: "A database management system (DBMS) organized around groups of records that share a common field value. Multidimensional databases are often generated from relational databases. Whereas relational databases make it easy to work with individual records, multidimensional databases are designed for analyzing large groups of records. The term OLAP (On-Line Analytical Processing) is become almost synonymous with multidimensional databases, whereas OLTP (On-Line Transaction Processing) generally refers to relational DBMSs". Từ định nghĩa ta rút ra được “multidimensional DBMS” gần nghĩa với "OLAP”, “multidimensional DBMS" là mở rộng của "relational databases" “multidimensional DBMS" có broader là "database management system".

Có những trường hợp sau khi phân tích mối quan hệ giữa hai keyphrases thì có thể xem như đồng nghĩa hoặc gần nghĩa. Và mối quan hệ gần nghĩa là sự bao hàm của mối quan hệ đồng nghĩa, có những mối quan hệ cũng được cung cấp sẵn tại Webopedia. Chẳng hạn ta xét thêm định nghĩa "tool” đã được cho cụ thể như sau: "A program that performs a very specific task. Synonymous with utility. Similar to application”. Như vậy dựa theo định nghĩa ta có thể xác định được mối quan hệ giữa program và utility là đồng nghĩa (synonym) và mối quan hệ giữa program và application là gần nghĩa (near-synonym).

Quy định về ký hiệu cho lớp (class) và keyphrases sử dụng: Theo các phân loại tại trang Webopedia về phân định phạm vi tin học, ta quy định tương ứng cho các lớp trong SPN-KOS nhưng sẽ được viết dưới dạng toàn bộ là chữ hoa (ví dụ: NGÔN NGŨ’ LẬP TRÌNH,...) và các keyphrases có thể có trường hợp ở dạng toàn bộ là chữ hoa nếu keyphrases đó là acronym của một keyphrases khác (ví dụ: keyphrases Ngôn ngũu đánh dấu siêu văn bản có acronym là HTML) hoặc ký tự đầu của keyphrases có thể là chữ hoa.

Biểu diễn keyphrases theo các quan hệ: Kết hợp với độ ưu tiên, cách lưu trữ các quan hệ giữa các keyphrases đề xuất ta xây dựng Ontology cho 3 lĩnh vực Phần mềm, Lập trình, Mạng máy tính. Đây là cơ sở để tiến hành tìm kiếm tài liệu theo nhu cầu thông 
tin của người dùng đưa vào. Từ danh sách các keyphrases của từng tài liệu cụ thể, ta tiến hành suy diễn ngữ nghĩa (Acronym, Synonym, Synonym, Broader, Narrower, Extenstion, Related) cho danh sách các keyphrases để phục vụ cho mục tiêu tìm kiếm theo ngữ nghĩa (thực hiện ở Bước 2 của mô hình nêu ở Mục 4.2.1). Hiệu năng của hệ thống tìm kiếm sẽ phụ thuộc vào kết quả của giai đoạn này.

Để đo lường mức độ liên quan của tài liệu, ta dùng trọng số cho mỗi keyphrase trong từng tài liệu cụ thể, độ ưu tiên càng cao thì mức độ liên quan càng lớn. Việc đánh trọng số ta dùng Lucene (như Bước 4 của mô hình ở Mục 4.2.1) cho từng keyphrases trên từng tài liệu cụ thể. Kết quả trả về danh sách tất cả các keyphrases trọng số của nó trong từng tài liệu cụ thể. Ta sẽ lưu các thông tin biểu diễn ngữ nghĩa vào một bảng dữ liệu để sử dụng cho giai đoạn thực thi tìm kiếm (thực hiện ở Bước 5 của mô hình nêu ở mục 4.2.1). Xây dựng Ontology (Inverted Index) tài liệu công nghệ thông tin trong phạm vi: Phần mềm; Lập trình; và Mạng máy tính.

\subsection{Kết quả thử nghiệm và đánh giá}

Ta tiến hành chạy thử nghiệm các mẫu ví dụ. Kết quả trả về tìm được hầu hết các tài liệu có liên quan đến nội dung cần tìm và được sắp xếp theo thứ tự độ quan trọng giảm dần (Hình 18).

Tuy nhiên, kết quả trả về trong tìm kiếm theo ngữ nghĩa với số lượng tài liệu rất lớn, các tài liệu có độ ưu tiên (điểm) rất thấp (được sắp xếp càng về sau) hầu như kém hoặc không liên quan gì đến nội dung cần tìm. Vì vậy để hiển thị kết quả trả về tối ưu, ta tiến hành thử nghiệm trên hai câu truy vấn cụ thể và tiến hành tính độ đo Recision, Recall thông qua hệ số đánh giá mức độ liên quan. Gọi $R$ là số lượng kết quả có liên quan trong kết quả truy tìm được; $T$ là tổng số văn bản truy tìm được; và $N$ là tổng số tài liệu liên quan trong hệ thống tài liệu. Tiến hành thử nghiệm các mẫu tìm kiếm tài liệu trên hệ thống SPN-KOS ta có kết quả như trong Bảng 2. 


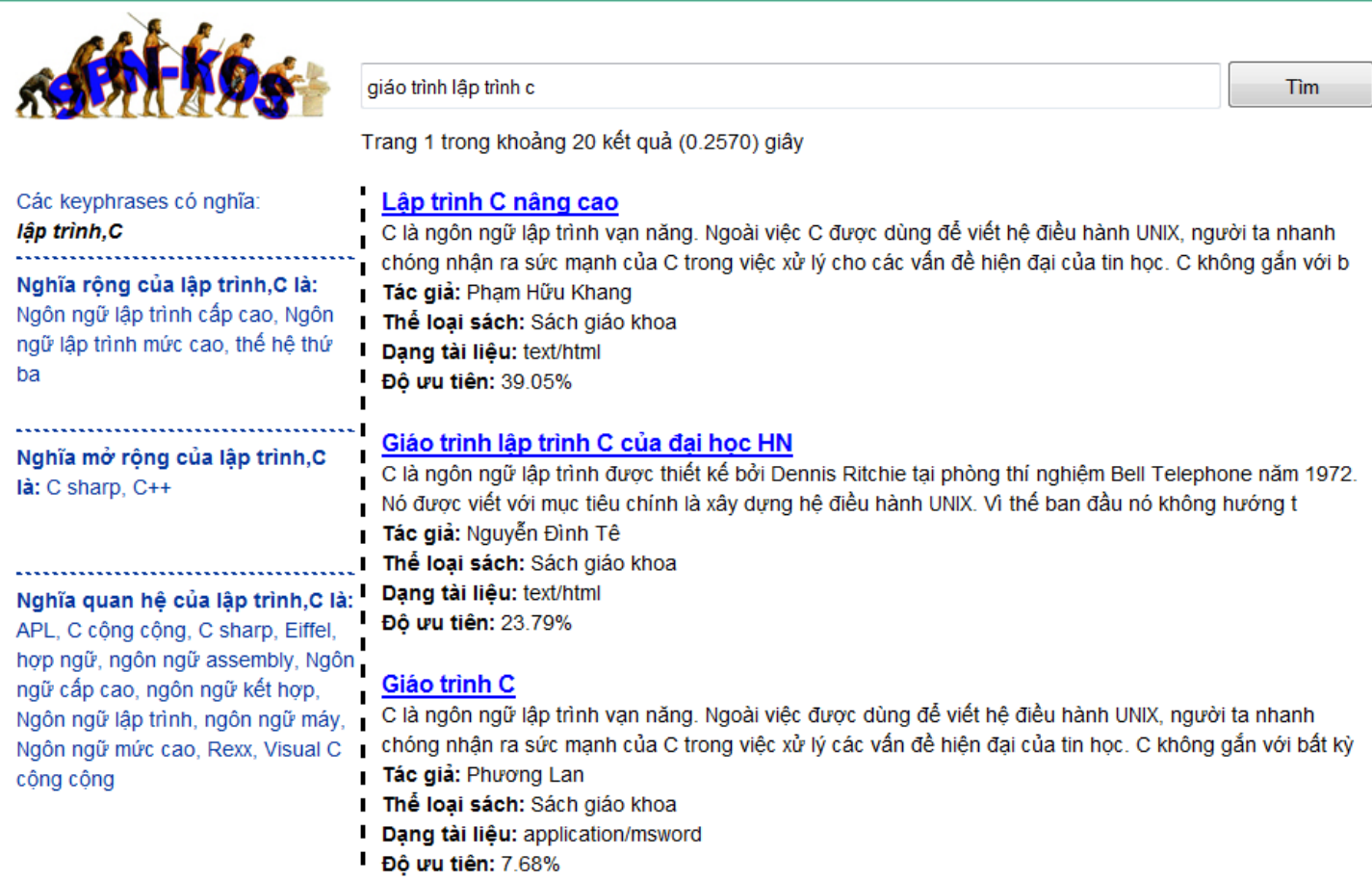

\section{Hình 18. Giao diện chương trình tìm kiếm văn bản theo ngữ nghĩa}

Từ Bảng 2, nhận thấy rằng các tài liệu ở phần đầu có độ chính xác cao hơn các tài liệu ở phần sau, còn giá trị độ bao phủ của tài liệu ở phần đầu có giá trị thấp hơn các tài liệu ở phần sau. Đồ thị đo độ chính xác và độ bao phủ của 2 câu truy vấn Q1, Q2 được biểu diễn như trong Hình 19.

Bảng 2. Độ chính xác và độ bao phủ

\begin{tabular}{lllll}
\hline & Q1 & \multicolumn{3}{c}{ Q2 } \\
\cline { 2 - 5 } & $\begin{array}{l}\text { Độ chính xác } \\
\text { Precison }(\mathrm{R} / \mathrm{T})\end{array}$ & $\begin{array}{l}\text { Độ bao phủ } \\
\text { Recall(R/N) }\end{array}$ & $\begin{array}{l}\text { Độ chính xác } \\
\text { Precison }(\mathrm{R} / \mathrm{T})\end{array}$ & $\begin{array}{l}\text { Độ bao phủ } \\
\text { Recall(R/N) }\end{array}$ \\
\hline Top 5 & $5 / 5=1.00$ & $5 / 22=0.23$ & $3 / 5=0.60$ & $3 / 9=0.33$ \\
Top 10 & $9 / 10=0.90$ & $9 / 22=0.41$ & $5 / 10=0.50$ & $5 / 9=0.56$ \\
Top 15 & $11 / 15=0.73$ & $11 / 22=0.50$ & $6 / 15=0.40$ & $6 / 9=0.67$ \\
Top 20 & $14 / 20=0.70$ & $14 / 22=0.64$ & $7 / 20=0.35$ & $7 / 9=0.78$ \\
Top 25 & $16 / 25=0.64$ & $16 / 22=0.73$ & $7 / 25=0.28$ & $7 / 9=0.78$ \\
Top 30 & $16 / 30=0.53$ & $16 / 22=0.73$ & $8 / 30=0.27$ & $8 / 9=0.89$ \\
\hline
\end{tabular}




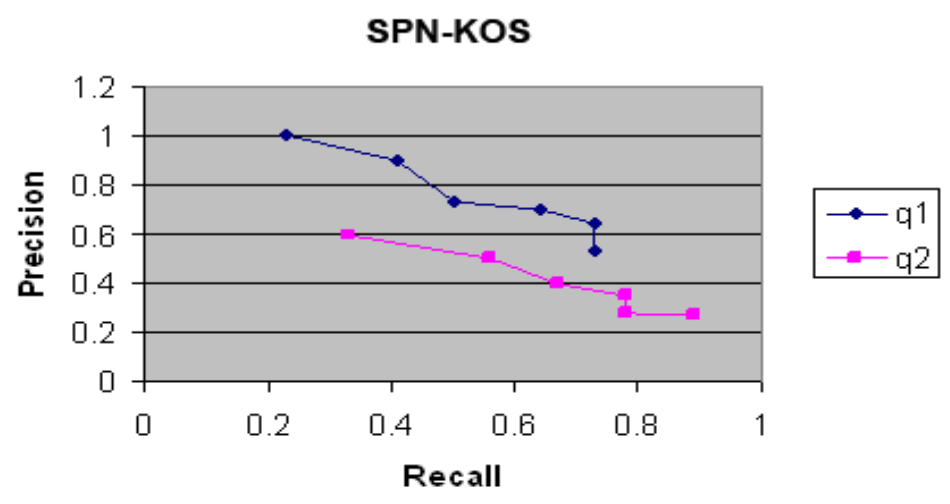

\section{Hình 19. Đồ thị độ chính xác và độ bao phủ}

Qua các mẫu thử nghiệm và dựa trên kết quả triển khai thử nghiệm, ta nhận xét thấy phương pháp quản lý, tìm kiếm theo ngữ nghĩa bước đầu đạt kết rất khả quan, đã giải quyết được khá tốt vấn đề tìm kiếm các văn bản liên quan đến nhau về mặt nội dung. Mục tiêu tìm kiếm văn bản theo ngữ nghĩa bước đầu thực hiện khá thành công. Tuy nhiên, phương pháp quản lý, tìm kiếm tài liệu theo ngữ nghĩa mất khá nhiều thời gian và chi phí cho việc xây dựng hệ trí thức (Ontology) cho một lĩnh vực, một ngành cụ thể. Chỉ có những chuyên gia trong ngành mới có thể thực hiện tốt được công việc này.

\section{KẾT LUẬN VÀ KIẾN NGH!}

Nghiên cứu này đã đưa ra giải pháp tìm kiếm nội dung tài liệu dựa trên ngữ nghĩa, xây dựng được kho dữ liệu thông dụng về công nghệ thông tin tiếng Việt trong các phạm vi: Phần mềm; Lập trình; và Mạng máy tính. Ngoài ra bài báo cũng đề xuất mô hình Ontology SPN-KOS cho cơ sở tài liệu văn bản có ngữ nghĩa cùng với kỹ thuật tổ chức lưu trữ và tìm kiếm theo ngữ nghĩa trong ba lĩnh vực: Phần mềm; Lập trình; và Mạng máy tính. Mô hình này nếu được mở rộng cho các lĩnh vực còn lại của công nghệ thông tin và các lĩnh vực khác sẽ mang lại một số ứng dụng như:

- Xây dựng một chương trình ứng dụng cụ thể phục vụ cho việc tìm kiếm theo ngữ nghĩa đáp ứng nhu cầu tìm kiếm tài liệu cho giảng viên và sinh viên;

- Xây dựng được Ontology SPN-KOS từ khối từ điển có thực về lĩnh vực công nghệ thông tin tiếng Việt trong phạm vi: Phần mềm; Lập trình; và Mạng máy 
tính;

- Biểu diễn cấu trúc thư mục có ngữ nghĩa dùng cho việc lưu trữ tài liệu, các tài liệu được phân định theo cấu trúc thư mục có ngữ nghĩa; Đã thử nghiệm trên kho tài liệu với khoảng vài trăm tài liệu và việc tìm kiếm theo ngữ nghĩa các tài liệu có liên quan ngữ nghĩa được trả về nhiều kết quả có liên quan đến nội dung cần tìm;

- Phương pháp tìm kiếm theo ngữ nghĩa có thể được xem là tiền đề thúc đẩy sự phát triển cho công nghệ thông tin về lĩnh vực tìm kiếm thông minh.

Để chương trình ứng dụng đi vào thực tế phục vụ cho việc nghiên cứu và học tập của giảng viên và sinh viên ngành công nghệ thông tin, ta nên tiếp tục nghiên cứu các phần việc sau:

- Tiếp tục mở rộng hệ thống ra các lĩnh vực còn lại của ngành công nghệ thông tin và các lĩnh vực khác;

- Nghiên cứu kỹ thuật để dữ liệu bên trong Ontology được cập nhật tự động để có nhiều tiện ích khi tra cứu thông tin;

- Phát triển các kỹ thuật học và khám phá tri thức dựa trên Ontology để tăng cường khả năng học và phát triển tri thức của các hệ tìm kiếm có ngữ nghĩa thông minh;

- Áp dụng mô hình để phát triển các sản phẩm phần mềm hoàn chỉnh có khả năng tìm kiếm theo song ngữ (Anh - Việt) trong đó cung cấp nhiều chức năng đa dạng được phân lớp để có thể dùng cho việc tìm kiếm các kiến thức - khái niệm - tài liệu ngữ nghĩa liên quan với nhiều mức độ khác nhau.

\section{TÀI LIỆU THAM KHẢO}

Aly, A. A. (2008). Using a query expansion technique to improve document retrieval. Information Technologies and Knowledge, 2(4), 343-348.

Cao, H. T. (2001). Nghiên cúu phát triển các kỹ thuật xây dụng và khai thác thông tin 
Web có ngũ nghĩa [Đề tài nghiên cứu cấp nhà nước]. Được truy lục từ http://lrc.tnu.edu.vn/upload/collection/brief/17620_6385.pdf

Christopher, D. M., Prabhakar, R., \& Hinrich, S. (2008). An introduction to information retrieval. Cambridge, England: Cambridge University Press.

David, I. (2008). Insight: A semantic file system [Technical report]. Retrieved from http:// www.dmi.me.uk/blog/wp-content/uploads/2008/01/insight-outsourcing.pdf

Lê, T. H., Từ, M. P., \& Huỳnh, Q. T. (2006). Tác tư công nghệ phần mềm hướng tác tủ. Hà Nội, Việt Nam: NXB Khoa học và Kỹ thuật

Lê, T. N. (2008). Xây dưng hệ thống tìm kiếm thông tin theo hướng tiếp cận ngũ nghĩa. (Luận văn Thạc sĩ), Trường Đại học Khoa học Tự nhiên Tp. Hồ Chí Minh, Việt Nam. Được truy lục từ http://doan.edu.vn/do-an/luan-van-xay-dung-he-tim-kiemthong-tin-theo-huong-tiep-can-ngu-nghia-conceptual-indexing-29630/

Nguyễn, T. M. T., \& Hoàng, H. H. (2009). Xây dựng Ontology cho thư viện số. Tạp chí Khoa hoc Đại hoc Huế, 9(53), 127-135.

Ngô, B. H. (2007). Xây dụng hệ thống tập tin ngũ nghĩa dựa trên bản thể học (Ontologybased Semantics File System) [Dự án Trường Đại học Cần Tho]]. Cần Thơ, Việt Nam: Trường Đại học Cần Thơ.

Roberto, B., Moschitti, A., Cammisa, M., Donati, E., \& Popov, B. (2006). Cross-language retrieval and access tools. Retrieved from http://prestospace.org/project/ deliverables/D16.3.pdf

Stokoe, C., Oakes, M. P., \& Tait, J. (2003). Word sense disambiguation in information retrieval revisited. Paper presented at The Annual ACM Conference on Research and Development in Information Retrieval, Canada.

Trương, C. L. (2009). Co sở tài liệu văn bản có ngũ nghĩa: Kỹ thuật tổ chíc luu trũ và tìm kiếm. TP. HCM, Việt Nam: Trường Đại học Công nghệ Thông tin.

Woods, W. A. (1997). Conceptual indexing: A better way to organize knowledge. Retrieved from http://dl.acm.org/citation.cfm?id=974965 


\title{
AN ONTOLOGY SEARCH FOR VIETNAMESE ACADEMIC DOCUMENTS IN COMPUTER SCIENCE
}

\author{
Nguyen Huu Vinh ${ }^{a^{*}}$
}

${ }^{a}$ The Faculty of Information Technology, Thudaumot University, Binhduong, Vietnam

"Corresponding author: Email: vinhnh@tdmu.edu.vn

\section{Article history}

Received: November $04^{\text {th }}, 2016 \mid$ Received in revised form: April 11 ${ }^{\text {th }}, 2017$

Accepted: May $11^{\text {th }}, 2017$

\begin{abstract}
Existing search engines were primarily designed to enhance keywork search, which provides little support for semantic meanings of a keyword query. This paper presents a semantic search tool based on ontological meanings for complete and effective searches. It proposes a conceptual model, database schema, and an instantiation of the tool. The proposed artefacts were evaluated using Vietnamese documents in three areas: Software engineering; Programming; and Computer networking. The evaluation results were presented and discussed.
\end{abstract}

Keywords: Ontology; Search engine; Semantic document. 